\title{
(5) \\ Evaluación formativa y formación integral de los estudiantes de educación básica
}

Formative evaluation and comprehensive training of basic education students

Avaliação formativa e formação integral de alunos do ensino básico

\author{
Juana Zambrano \\ Universidad César Vallejo, Perú \\ https://orcid.org/0000-0002-5393-0530 \\ Cecilia Mendoza-Alva \\ Universidad César Vallejo, Perú \\ https://orcid.org/0000-0002-3640-2779
}

DOI: https://doi.org/10.35622/j.rep.2021.03.001

Recibido 13/12/2020/ Aceptado 20/07/2021

RESUMEN. La presente investigación tiene como finalidad determinar cómo influye la evaluación formativa en la formación integral de los estudiantes de la Institución Educativa № 80055 "Juan Ignacio Gutiérrez Fuente”, Macabí Bajo, Paiján, 2020. Considerando un método de investigación cuantitativo de tipo no experimental y diseño descriptivo correlacional causal. La muestra estuvo conformada por 28 docentes elegidos a través del muestro no probabilístico. La técnica fue la encuesta y sus instrumentos empleados un cuestionario para analizar la evaluación formativa y un cuestionario para evaluar la formación integral, analizados por el estadígrafo Alpha de Cronbach y validados mediante juicio de expertos para el análisis de procesamiento de datos, se aplicó la estadística descriptiva e inferencial usando la prueba de correlación de Rho de Spearman. Los resultados demuestran que evaluación formativa influye significativamente en la formación integral de los estudiantes de la Institución Educativa. Se obtuvo un Rho Spearman= 0.737 y el significado bilateral obtenido es 0.001 valor que es inferior a la región crítica $\alpha=0.05$, en consecuencia, se rechaza la hipótesis nula y se acepta la hipótesis alterna que concluye que la evaluación formativa influye significativamente en la formación integral de los estudiantes de la Institución Educativa № 80055.

PALABRAS CLAVE: aprendizaje, evaluación formativa, evidencias, formación integral, retroalimentación.

ABSTRACT. The purpose of this research is to determine how formative evaluation influences the integral formation of the students of the Educational Institution N 80055 "Juan Ignacio Gutiérrez Fuente", Macabí Bajo, Paiján, 2020. Considering a non-experimental quantitative research method and causal correlational descriptive design. The sample consisted of 28 teachers chosen through non-probabilistic sampling. The technique was the survey and its instruments used a questionnaire to analyze the formative evaluation and a questionnaire to evaluate the integral formation, analyzed by Cronbach's Alpha statistician and validated by an expert judgment for the analysis of data processing, the statistics were applied descriptive and inferential using Spearman's Rho correlation test. The results show that formative evaluation significantly influences the integral formation of the students of the Educational Institution. A Rho Spearman $=0.737$ was obtained and the bilateral meaning obtained is 0.001 value that is lower than the critical region $\alpha=0.05$, consequently, the 
null hypothesis is rejected and the alternative hypothesis is accepted, which concludes that the formative evaluation significantly influences the comprehensive training of the students of the Educational Institution N ${ }^{\circ} 80055$

KEYWORDS: learning, formative evaluation, evidence, comprehensive training, feedback

RESUMO. 0 objetivo desta pesquisa é determinar como a avaliação formativa influencia a formação integral dos alunos da Instituição de Ensino N 80055 "Juan Ignacio Gutiérrez Fuente", Macabí Bajo, Paiján, 2020. Considerando um método de pesquisa quantitativa não experimental e correlacional causal design descritivo. A amostra foi composta por 28 professores escolhidos por meio de amostragem não probabilística. A técnica foi a survey e seus instrumentos utilizaram um questionário para análise da avaliação formativa e um questionário para avaliação da formação integral, analisado pelo estatístico Alfa de Cronbach e validado por julgamento de especialistas para a análise do processamento dos dados, as estatísticas foram aplicadas descritiva e inferencial utilizando Teste de correlação Rho de Spearman. Os resultados mostram que a avaliação formativa influencia significativamente na formação integral dos alunos da Instituição de Ensino. Obteve-se Rho Spearman $=0,737$ e o significado bilateral obtido é 0,001 valor inferior à região crítica $\alpha=0,05$, conseqüentemente, rejeita-se a hipótese nula e aceita-se a hipótese alternativa, o que conclui que a avaliação formativa influencia significativamente 0 abrangente treinamento dos alunos da Instituição Educacional $N{ }^{\circ} 80055$.

PALAVRAS CHAVE: aprendizagem, avaliação formativa, evidências, treinamento abrangente, feedback.

\section{INTRODUCCIÓN}

El proceso de la evaluación es un tema de importancia dentro de las instituciones educativas donde los docentes comprueban el grado de adquisición de aprendizajes de los estudiantes según los estándares planteados en el currículo nacional de cada país; así mismo es un proceso que se realiza de manera sistemática e integrada donde se obtiene información relevante que orienta el trabajo más distante de la medición u obtención de puntajes, para luego tomar decisiones que permitan ayudar, mejorar y ajustar el accionar educativo. Por otra parte, la formación integral se concibe como un proceso donde se desarrollan las potencialidades de los estudiantes desde las actitudes, capacidades y competencias que les permiten actuar de manera ética y responsable en cualquier situación de la vida. A nivel de Latinoamérica se busca mejorar el nivel educativo mediante el desarrollo de sistemas de evaluación que admitan no solamente obtener calificativos de forma numérica sino brindar insumos para la mejora del proceso dentro de las aulas de clase (Pigott y Polanin, 2011). Es necesario mencionar que años atrás solo se centraba la atención en la nota que obtenía el estudiante sin importar como lo adquiría, a esto se suma que se practicaba una educación tradicional, donde la construcción de los aprendizajes se realizaba de manera conductual penalizando los malos resultados y premiando los buenos. Además, las evaluaciones estandarizadas como PISA (2018) en donde los países de América Latina participan han obtenido puntajes bajos comparado con los países europeos o asiáticos. Por eso, actualmente están convencidos de la importancia de medir el rendimiento académico 
identificando aquellos obstáculos y logros de los estudiantes para empezar a construir aprendizajes significativos; por lo cual se ha producido cambios desde la forma de enseñar y aprender hasta llegar a una evaluación formativa, la cual consiste en la regulación del proceso de enseñanza como consecuencia del recojo de información que consiga la toma de decisiones mediante la reflexión que se realiza entre el estudiante y docente en el recojo de evidencias. Por otra parte, la formación integral de los estudiantes es influenciada por aspectos como el inadecuado manejo de la tecnología, las malas relaciones dentro de la familia, la influencia social de la moda, música y modos de vivir, las estrategias de enseñanza, los planes de estudio y las políticas educativas, entre otros son aspectos que no permite que los estudiantes de educación básica puedan lograr un desarrollo adecuado en su formación integral. Uno de los países que ha puesto énfasis y preocupación en el desarrollo de sus estudiantes es Finlandia, quien invierte en educación a través de políticas que tienen como centro al niño y adolescente.

A nivel nacional, el Ministerio de Educación (2016) mediante una reforma educativa busca el desarrollo de capacidades que permitan al estudiante enfrentar la vida a través del despliegue de sus competencias, capacidades, actitudes y valores, es por ello que la evaluación formativa debe orientar un proceso ordenado donde se obtienen evidencias y se realiza una valoración al estudiante para identificar el nivel de logro alcanzado en la competencia trabajada. Resulta necesario que se efectúe un proceso de capacitación para que los docentes a nivel nacional se empoderen de este tipo de evaluación dejando de lado el proceso de mediación tradicional para conseguir que el estudiante obtenga una formación integral. Asimismo, es necesario tener presente que por muchos años en el Perú se ha manejado, propuesto y ejecutado modelos educativos de otros países que no se han sostenido en el tiempo y no han permitido obtener buenos resultados porque no responden a las características de los educandos, es por eso que en estos últimos años se busca que el estudiante pueda tener una formación integral donde se involucre el saber ser, el hacer, vivir y convivir; pero es necesario que uno de los agentes principales (docentes) puedan estar capacitados para realizar una enseñanza conforme a los avances de la ciencia y tecnología, así como los requerimientos de estos últimos tiempos globalizados para formar al estudiante de manera integral, la cual ha sido perjudicada por influencia del medio social, la moda, ideologías asociadas a pensamientos radicales, adquisición de modelos educativos 
de otros países que no se ajustan a las características de los niños y adolescentes del Perú. A esto se suma la corrupción, la violencia en las calles, el clima social familiar, entre otros que son aspectos que deben de tenerse en cuenta para conseguir en los estudiantes una formación integral.

A nivel de la Institución Educativa № 80055 “Juan Ignacio Gutiérrez Fuente", Macabí Bajo, Paiján se observa que los docentes presentan dificultades para realizar el proceso de evaluación porque aún no se tiene un buen nivel de conocimiento de cómo ejecutarlo para la obtención de los logros de los estudiantes, según las competencias trabajadas; lo cual influye de manera directa en la formación integral de los estudiantes porque la evaluación no se realiza de manera correcta, no se conoce que capacidades se deben de retroalimentar; a esto se suma que el medio familiar donde se desenvuelven los estudiantes en muchos casos no es el adecuado y esto influye en su formación académica. Además, con respecto a la formación integral de los estudiantes se evidencia que viene siendo influenciada por aspectos como la falta de valores para el cumplimiento de los deberes y respeto de las diferencias, no practican el compañerismo al realizar trabajos en equipo, no presentan un pensamiento crítico - reflexivo, tienen dificultades para la resolución de conflictos, no han desarrollado capacidades como la autonomía y resiliencia, valores morales y espirituales, entre otros. Por tal motivo se ha determinado trabajar el siguiente problema de investigación: ¿En qué medida la evaluación formativa influye en la formación integral en los estudiantes de la Institución Educativa N 80055 "Juan Ignacio Gutiérrez Fuente", Macabí Bajo, ¿Paiján?

La realidad problemática se aborda mediante el proceso de la observación donde se identificaron una variedad de problemas, pero el presente tiene mayor relevancia. Luego de haber escogido las variables a estudiar, se procedió con la construcción de la parte cualitativa, se escogió el diseño de investigación la cual permitió recoger las principales características del objeto de estudio; a partir de dicha información se realizó el procedimiento estadístico para determinar el nivel de influencia entre las variables consideradas en el estudio. La investigación reviste conveniencia porque permitió conocer aspectos importantes de las variables de estudio para determinar si existe influencia entre ellas a través del empleo de instrumentos como cuestionarios que brindaron resultados 
válidos y confiables. Se convertirá en un trabajo previo que puede ser consultado por investigadores de diferentes ámbitos. Tiene relevancia social porque se trabajó con un equipo humano parte de la muestra de estudio a quienes se les aplicó los cuestionarios que permitieron determinar el nivel de ambas variables para luego entregar los resultados a las autoridades de la institución y contribuir con un trabajo experimental en un futuro no lejano para mejorar y fortalecer el comportamiento de ambas variables. Así mismo, presentó implicancia practica porque se conoció la influencia que existe entre evaluación formativa y formación integral que se convertirá en antecedentes a futuros investigadores para la ejecución de indagaciones con mayor profundidad. Además, los instrumentos han sido creados por la investigadora los cuales han sufrido un proceso de validación y confiabilidad para ser aplicado a la muestra de estudio. Tiene valor teórico porque se procedió a buscar información en fuentes confiables para la construcción del marco teórico, legal, histórico y fisiológico sobre las variables y sus dimensiones a través de un análisis y sistematización de la información teniendo en cuenta aportes de autores e investigadores. Su utilidad metodológica se realizó a través de transportar a las variables de un proceso complejo a uno simple a mediante la operacionalización y se seleccionó un diseño de investigación que permitió la recolección de los datos.

Los trabajos previos que se relacionan con las variables de la presente investigación son los siguientes: A nivel internacional, Rodríguez (2017) en su tesis: "Evaluación de un currículo centrado en la formación integral de los estudiantes", Universidad Católica, Colombia, de tipo cualitativa, realizada mediante un estudio de casos donde participaron 35 sujeto muéstrales a quienes se les aplicó cuestionarios, concluye que el nivel de satisfacción en cuanto al perfil del docente y el sistema de evaluación que se emplea se ubican en el nivel de satisfactorio. Existe un adecuado nivel en el empleo de estrategias para la formación integral de los educandos, se evidencia un adecuado nivel de contextualización del currículo en un evidente desarrollo de valores que se encuentran determinados según el diagnóstico del PEl lo cual se traduce en la búsqueda de la eliminación de las debilidades. Por su parte Vera (2019) es investigación de tipo no experimental "Evaluación formativa y la producción de textos escritos de los estudiantes del V ciclo de la I.E. № 20547 Manuel A. Odría de la Ugel 15 - Huarochirí, Universidad César Vallejo", con el objetivo de determinar la relación que existe entre las variables, utilizo el diseño descriptivo - correlacional en 470 niños y 150 
jóvenes a quienes se les aplicó cuestionarios, concluyendo que existe una relación positiva de Rho $=0,920 p_{\text {_ }}$ valor $=0,000<0,005$ por lo tanto, se determina que la evaluación formativa tiene una relación significativa en la elaboración de textos de manera escrita. Un 79\% de estudiantes mencionan que la evaluación formativa que aplica su docente es adecuada y destacada. Además, Paredes (2019) en su investigación "Gestión tutorial y formación integral del estudiante de educación inicial de la Institución Educativa Salaverry2019" tuvo por objetivo identificar la relación que existe entre las variables, de tipo no experimental y diseño correlacional, realizado en una muestra conformada por 30 padres de familia, a quienes se les aplicó cuestionarios, concluye que existe una relación positiva de Rho $=0.630$ entre las variables de estudio, lo cual demuestra que una guarda relación con la otra pero que existen otros factores que alteran su comportamiento.

A continuación, se procede al desarrollo de las teorías de la variable evaluación formativa según Condemarín y Medina (2012) es el tipo de evaluación que permite el logro de aprendizaje adquirido por el estudiante, proyectándose hacia donde más puede llegar a través de un proceso de retroalimentación, donde se regula y reflexiona sobre los logros obtenidos de acuerdo a los estándares establecidos utilizando estrategias que de acuerdo las necesidades de los estudiantes. Por su parte, el Ministerio de Educación (2016) la evaluación formativa según el CNEB es un proceso que tiene como objetivo garantizar la formación integral del estudiante a través de acompañamiento sistemático, regulado y persistente se logra lo planteado teniendo en cuenta los objetivos educativos que se desarrolla a través de acciones, que van más allá de la obtención de sólo calificaciones, dejando de lado lo correcto e incorrecto, sino la reflexión de lo obtenido. Así mismo sus dimensiones son según William (2011) la dimensión clasificar metas de aprendizaje, que se refiere al proceso de la presentación, aclaración y comprensión de los propósitos de aprendizaje que deben de adquirir los estudiantes para la construcción de las competencias según lo planteado en el currículo nacional; teniendo en cuenta que para la obtención de las metas es necesario tener claro lo que se quiere lograr. La dimensión obtener evidencias consiste en realizar un conjunto de acciones para realizar el proceso de recopilación de la información entendible y manejable que pueda ser empleada para mejorar el proceso de enseñanza. La dimensión retroalimentación se refiere al proceso de recoger de los estudiantes sus evidencias de aprendizaje para determinar cuál es su desenvolvimiento en 
las actividades asignadas para poder realizar el proceso de autorregulación donde se produzca la reflexión y la mejora según las observaciones realizada por el docente. La dimensión, estudiantes como dueños de su aprendizaje, se considera como un aspecto fundamental dentro del proceso de evaluación formativa, porque en ellos se centra el desarrollo de la enseñanza según los objetivos propuestos. Para observar dicho protagonismo es necesario que convierta en el constructor de su propio aprendizaje a través de un protagonismo activo dentro de las aulas de clase. La última dimensión, estudiantes como recursos de aprendizaje se traduce como, los agentes en donde el docente construye competencias y por lo cual lo considera como recursos, además se convierten en un medio de aprendizaje cuando comparten sus saberes con sus compañeros. Además, esta estrategia es un punto de inicio para combinar las otras dimensiones ya trabajadas.

Scriven (1967) sostiene que existen 2 tipos de evaluaciones, una es la que solo tiene como objetivo la calificación de los logros obtenidos por los estudiantes y la otras consisten en un proceso donde se tiene en cuenta el proceso de enseñanza aprendizaje donde se tiene en cuenta los avances y necesidades de los estudiantes. De la misma manera Sadler (1989) manifiesta que la información obtenida a través de la evaluación formativa sirve tanto para el docente para determinar que estrategias puede utilizar para contribuir con el desarrollo de las competencias de los estudiantes y sirve para el educando porque le permite identificar cuales fueron aquellas falencias dentro de su evidencia entrega, de manera que pueda corregirla y mejorarla a través de un proceso de retroalimentación. También Brookhart (2003) señala que este tipo de evaluación tiene en cuenta aspectos cognitivos de los estudiantes como aquellos que lo estimulan para brindarle a los estudiantes una información precisa de aquellos aprendizajes que se necesitan mejorar, teniendo en cuenta un proceso que no dañe las emociones de los estudiantes, sino que les permita ser conscientes de su aprendizaje a través de un autocontrol sobre los logros obtenido. Existen teorías contemporáneas del aprendizaje que involucran la evaluación formativa que se ajusta y es compatible con teorías cognitivas y socioculturales que sustentan el currículo nacional en el Perú; por su parte la teoría cognitiva, busca que el aprendizaje nuevo se relacione con los aprendizajes previos del estudiante y la teoría sociocultural, sostiene que el aprendizaje se desarrolla mejor cuando es en conjunto o en grupo, vinculados con su cultura (Shepard, 2006). Así mismo, el sustento teórico de la variable formación integral donde Paiba (2007) 
sostiene que se traduce cuando se desarrollan todas las dimensiones en un ser humano (cognitivo, actitudinal, habilidades y valores) empezando desde el aspecto intelectual a través de la construcción de aprendizajes, el aspecto humano adquiriendo valores morales y la ética, así como el aspecto social para realizar una interacción saludable.

Las dimensiones de la variable formación integral fueron plateadas por el MINEDU (2016) las cuales son dimensión humana, que consiste en desarrollar las capacidades, virtudes y actitudes que están relacionadas con llevar una vida que respeta la condición humana y el entorno social donde se desenvuelve, es necesario saber que el ser humano presenta dos facultades que lo diferencian de los animales irracionales, las cuales son la inteligencia para decidir y actuar correctamente y la voluntad que los hacen personas racionales y superiores.

La dimensión intelectual según está traducida en el desarrollo del pensamiento lógico, critico, analítico y creativo que le permita la obtención de aprendizaje significativos y de tener una autoformación a lo largo de su vida; de esta manera el estudiante es competente, adquiere habilidades para razonar, analizar, argumentar, entre otros que le permita generar nuevos conocimientos y brindar soluciones creativas y precisas a los problemas que se presentan. Dimensión axiológica sostienen que es necesario que se adquieran valores que construyan su personalidad, les permita construir sus percepciones y tomar decisiones; es necesario sostener que los seres humanos somos diferentes, por ende, es necesario que nos respetemos para tener una mejor convivencia. Dimensión social sostiene que para que el ser humano vida en sociedad necesita fortalecer sus valores y actitudes que le permitan respetar y valorar al otro; por eso, es necesario que el ser humano sea sensible, reconozca las problemáticas sociales para brindarle soluciones a través de trabajos de manera individual o grupal, respetando opiniones y la diversidad cultural. La teoría que sustenta la formación integral es la de Maslow (1943), quien señala que existe una jerarquía de necesidades que deben de ser satisfechas durante todo el proceso de crecimiento como persona. De acuerdo a como el ser humano empieza a satisfacer sus necesidades básicas van a aparecer otras en su actuar diario que condicionan y cambian el comportamiento, por ella plantea una categoría de necesidades de acuerdo a las prioridades. La investigación presenta un marco legal porque se sustenta en la ley general de educación $N^{\circ} 28044$, que sostiene en su artículo 22, que el proceso de la evaluación que se realiza en las instituciones 
educativas tiene como objetivo principal la identificación de los logros obtenidos por los estudiantes en las diferentes áreas pedagogías, con la finalidad brindarle al educando el apoyo correspondiente para que pueda mejorar el desarrollo de sus competencias, capacidades y actitudes que se han establecido en el marco curricular a través de sus estándares básicos. Asimismo, se sostiene que el Ministerio de Educación es el encargado y responsable de establecer los criterios para la evaluación, promoción de grado, recuperación y las nivelaciones académicas que se requieran de acuerdo a la diversidad de los estudiantes. Además, sostiene el derecho a la educación se debe de dar a lo largo de la vida y que debe de contribuir a su formación integral, desarrollando sus potencialidades, aspectos importantes de la cultura, la familia y la comunidad nacional, de América Latina y el mundo. Su marco histórico sostiene que la evaluación en el ámbito educativos ha sufrido un proceso constante de cambios, desde Tylor en el periodo pretyeriano, fue el primero en quien cogió la palabra evaluación, luego se pasó con las practicas chicas para seleccionar su personal en el siglo II, quienes utilizaron la evaluación; seguido en el siglo V, se crearon los famosos cuestionarios, luego surgen las universidades, viene la didáctica magna de Juan Amos Commenio, más tarde en el siglo XIX conocimos a la escuela tradicional en donde se evaluaba la retención de conocimientos, se conocen los diferentes tipos de evaluación, hasta llegan en la actualidad donde no se realiza un simple proceso de medición, sino una evaluación formativa que le permita al docente reconocer competencias adquiridas y debilidades para el proceso de retroalimentación. Así mismo, desde épocas muy antiguas se busca que la persona presente un alto nivel de formación, para que pueda enfrentar los desafíos de la vida, por eso desde tiempos muy antiguos se han creado leyes como el derecho a la educación gratuita para que los niños desde pequeños puedan ser formados desde diferentes dimensiones para obtener una formación integral. Presenta un marco filosófico, porque se busca que el ser humano tenga la capacidad de reflexionar sobre su propio actuar, donde los profesionales comprenden el sentido e implicancias antropológicas y éticas sobre su actuación diaria para la formación integral de estudiantes a través de una práctica pedagógica adecuada, con un alto nivel de conocimiento. A esto, se puede agregar la filosofía de la evaluación lo cual contempla un saber en acción, para la acción y desde la acción; es decir implica una evaluación integral de la persona a través de reflexiones imparciales (Amilburu 2010). 


\section{MÉTODO Y MATERIALES}

La investigación presenta un enfoque metodológico, de tipo básico, no experimental, con diseño descriptivo correlacional - causal, porque se busca determinar la influencia que existe entre las variables estudiadas (Hernández, Fernández y Baptista, 2006). El siguiente grafico corresponde a este tipo de diseño:

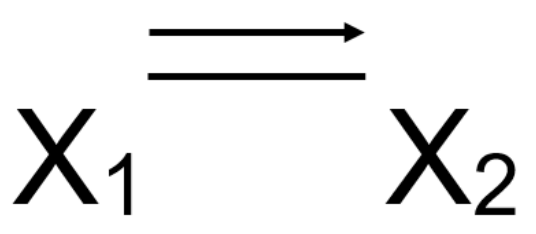

Donde:

$\mathrm{X}_{1}=$ Variable 1: Evaluación formativa

$\mathrm{X}_{2} \quad=$ Variable 2: Formación integral

$\vec{I}$ Influencia de $X_{1}$ sobre $X_{2}$

$=$ Relcion entre las variables.

La población se considera como conjunto de individuos, objetos, situaciones, etc., de quienes el investigador desea identificar su comportamiento, las cuales se convierten en unidades observables para el recojo de información que permite llegar a conclusiones (Velásquez, 1999). La población en la presente investigación estuvo constituida por los docentes del nivel primario y secundario de la I.E. N 80055 “Juan Ignacio Gutiérrez Fuente”, Macabí Bajo, Paiján. La muestra en la presente investigación estuvo constituida por 28 docentes del nivel primario y secundario de la Institución Educativa en estudio. El tipo de muestreo utilizado es el no probabilístico, es decir no se encoje de manera aleatoria sino en función de su disponibilidad. Los cuestionarios se emplean en las investigaciones experimentales y no experimentales para la recolección de datos, elaborados de forma confiable y rigurosa teniendo en consideración lo que se desea medir en la indagación y son útiles para realizar la descripción del análisis estadístico descriptivo e inferencial. Para medir la variable evaluación formativa se generó un instrumento que es un cuestionario que contiene 20 ítems elaborado teniendo en cuenta sus subvariables: clasificar metas de 
aprendizaje, obtener evidencias, retroalimentación, estudiantes como dueños de su aprendizaje y estudiantes como recursos de aprendizaje cada una con 4 preguntas, las cuales se pueden responder con siempre, a veces y nunca. Las dimensiones del cuestionario han sido propuestas por William (2011).

Para medir la variable formación integral se generó un instrumento de recolección de datos consistente en un cuestionario que contiene 20 preguntas elaborado en función de sus dimensiones dimensión humana, dimensión intelectual, dimensión axiológica y dimensión social cada una con 5 preguntas las cuales se pueden responder marcando las opciones de respuesta siempre, a veces y nunca. Las dimensiones del cuestionario han sido propuestas por MINEDU (2016). Una vez construido los cuestionarios se solicitó el permiso correspondiente al director de la institución educativa a través de una solicitud para la aplicación de los instrumentos creados que miden ambas variables, luego se concientizó a los docenes pertenecientes a la muestra de estudio para que puedan colaborar con el trabajo de campo a través de un sinceramiento en cada respuesta otorgada. Así mismo, al momento de la aplicación del instrumento se realizó a través de diversos medios y momentos donde los docentes tenían un tiempo prudencial para responder sin ningún tipo de distracción o manipulación. Una vez obtenido los datos se realizó el procesamiento estadístico empleando los programas de SPSS-25 y Excel 2016 para la creación de tablas y figuras estadísticas, la obtención de la prueba de normalidad que determinó la prueba de hipótesis a emplear. Por último, se realizó la discusión de los resultados a través de la triangulación entre los datos obtenido, la contrastación con los trabajos previos y el sustento con el marco teórico para luego terminar con la formulación de las conclusiones y recomendaciones.

\section{RESULTADOS}

\section{Tabla 1}

Nivel de evaluación formativa en los docentes de la Institución Educativa N 80055 "Juan Ignacio Gutiérrez Fuente", Macabí Bajo, Paiján, 2020.

\begin{tabular}{cccc}
\hline & & $(\mathrm{f})$ & $(\%)$ \\
\hline \multirow{3}{*}{ Válido } & Bajo & 2 & $7,1 \%$ \\
& Medio & 18 & $64,3 \%$ \\
& Alto & 8 & $28,6 \%$ \\
\hline \hline
\end{tabular}


Total 28

$100,0 \%$

Nota. Data de resultados obtenidos.

En la tabla se observa los resultados de las variables evaluación formativa obtenidos luego de la aplicación del cuestionario a los docentes de la Institución Educativa № 80055 "Juan Ignacio Gutiérrez Fuente", Macabí Bajo, Paiján, 2020. Se observa que el $7,1 \%$ de los docentes presentan un bajo nivel de evaluación formativa; el 64,3\% de los docentes se ubican en el nivel medio y el 28,6\% presentan un alto nivel de evaluación formativa.

\section{Tabla 2}

Nivel de percepción de la formación integral de los estudiantes en los docentes de la Institución Educativa No 80055 “Juan Ignacio Gutiérrez Fuente”, Macabí Bajo, Paiján, 2020.

\begin{tabular}{cccc}
\hline & & $(\mathrm{f})$ & $(\%)$ \\
\hline \multirow{3}{*}{ Válido } & Bajo & 2 & $7,1 \%$ \\
& Medio & 20 & $71,5 \%$ \\
& Alto & 6 & $21,4 \%$ \\
& Total & 28 & $100 \%$ \\
\hline \hline
\end{tabular}

Nota. Data de resultados obtenidos.

En la tabla se observa los resultados de las variables evaluación formativa obtenidos luego de la aplicación del cuestionario a los docentes de la Institución Educativa № 80055 "Juan Ignacio Gutiérrez Fuente", Macabí Bajo, Paiján. Se observa que el 7,1\% de los docentes perciben un bajo nivel de formación integral de los estudiantes; el 71,5\% de los docentes se ubican en el nivel medio y el $21,4 \%$ perciben un alto nivel de formación integral de los estudiantes.

\section{Tabla 3}

Niveles de las dimensiones de la variable evaluación formativa en la Institución Educativa $N^{\circ}$ 80055 “Juan Ignacio Gutiérrez Fuente”, Macabi Bajo, Paiján, 2020.

\begin{tabular}{lcccccccc}
\hline \multirow{2}{*}{ Variable / Dimensiones } & \multicolumn{3}{c}{ Bajo } & \multicolumn{3}{c}{ Medio } & \multicolumn{3}{c}{ Alto } & Total \\
\cline { 2 - 7 } & $\mathrm{n}$ & $\%$ & $\mathrm{n}$ & $\%$ & $\mathrm{n}$ & $\%$ & \\
\hline D1. Clasificar metas de aprendizaje & 3 & $10,7 \%$ & 13 & $46,4 \%$ & 12 & $42,9 \%$ & 28 \\
D2. Obtener evidencias & 4 & $14,3 \%$ & 13 & $46,4 \%$ & 11 & $39,3 \%$ & 28 \\
D3. Retroalimentación & 4 & $14,3 \%$ & 15 & $53,6 \%$ & 9 & $32,1 \%$ & 28 \\
D4. Estudiantes como dueños de su aprendizaje & 5 & $17,9 \%$ & 15 & $53,6 \%$ & 8 & $28,6 \%$ & 28 \\
D5. Estudiantes como recursos de aprendizaje. & 6 & $21,4 \%$ & 8 & $28,6 \%$ & 14 & $50,0 \%$ & 28 \\
\hline
\end{tabular}

Nota. Data de resultados obtenidos. 
En la tabla se observa los resultados de las dimensiones de la variable evaluación formativa obtenidos luego de la aplicación del cuestionario a los docentes de la Institución Educativa N 80055 “Juan Ignacio Gutiérrez Fuente”, Macabí Bajo, Paiján. Se observa que el 10,7\% de los docentes presentan un bajo nivel para clasificar metas de aprendizaje; el 46,4\% de los docentes se ubican en el nivel medio y el 42,9\% presentan un alto nivel para clasificar metas de aprendizaje. Además, el 14,3\% de los docentes presentan un bajo nivel para obtener evidencias; el 46,4\% de los docentes se ubican en el nivel medio y el 39,3\% presentan un alto nivel para obtener evidencias. Así mismo, el 14,3\% de los docentes presentan un bajo nivel para realizar la retroalimentación; el 53,6\% de los docentes se ubican en el nivel medio y el 32,1\% presentan un alto nivel para realizar la retroalimentación.

Por otra parte, el 17,9\% de los docentes perciben un bajo nivel de estudiantes como dueños de su aprendizaje; el 53,5\% de los docentes se ubican en el nivel medio y el 28,6\% perciben un alto nivel de estudiantes como dueños de su aprendizaje. Por último, el 21,4\% de los docentes perciben un bajo nivel de estudiantes como recursos de aprendizaje; el 28,6\% de los docentes se ubican en el nivel medio y el 50,0\% perciben un alto nivel de estudiantes como recursos de aprendizaje.

\section{Tabla 4}

Niveles de las dimensiones de la variable formación integral de los estudiantes de la Institución Educativa N 80055 “Juan Ignacio Gutiérrez Fuente”, Macabí Bajo, Paiján, 2020.

\begin{tabular}{lccccccc}
\hline \multirow{2}{*}{ Variable / Dimensiones } & \multicolumn{3}{c}{ Bajo } & \multicolumn{2}{c}{ Medio } & \multicolumn{3}{c}{ Alto } & \multirow{2}{*}{ Total } \\
\cline { 2 - 7 } & $\mathrm{n}$ & $\%$ & $\mathrm{n}$ & $\%$ & $\mathrm{n}$ & $\%$ & \\
\hline Dimensión humana & 6 & $21,4 \%$ & 13 & $46,4 \%$ & 9 & $32,1 \%$ & 28 \\
Dimensión intelectual & 6 & $21,4 \%$ & 14 & $50,0 \%$ & 8 & $28,6 \%$ & 28 \\
Dimensión axiológica & 7 & $25,0 \%$ & 15 & $53,6 \%$ & 6 & $21,4 \%$ & 28 \\
Dimensión social & 3 & $10,7 \%$ & 19 & $67,9 \%$ & 6 & $21,4 \%$ & 28 \\
\hline
\end{tabular}

Nota. Data de resultados obtenidos.

En la tabla se observa los resultados de las dimensiones de la variable formación integral obtenidos luego de la aplicación del cuestionario a los docentes de la Institución Educativa N 80055 "Juan Ignacio Gutiérrez Fuente”, Macabí Bajo, Paiján, 2020. Se observa que el 21,4\% de los docentes perciben un bajo nivel de la dimensión humana de la formación 
integral; el $46,5 \%$ de los docentes se ubican en el nivel medio y el $32,1 \%$ perciben un alto nivel de la dimensión humana de la formación integral. Además, el 21,4\% de los docentes perciben un bajo nivel de la dimensión intelectual de la formación integral; el 50,0\% de los docentes se ubican en el nivel medio y el 28,6\% perciben un alto nivel de la dimensión intelectual de la formación integral. Por otra parte, el 25,0\% de los docentes perciben un bajo nivel de la dimensión axiológica de la formación integral; el 53,6\% de los docentes se ubican en el nivel medio y el 21,4\% perciben un alto nivel de la dimensión axiológica de la formación integral y por último, el 10,7\% de los docentes perciben un bajo nivel de la dimensión social de la formación integral; el 67,9\% de los docentes se ubican en el nivel medio y el 21,4\% perciben un alto nivel de la dimensión social de la formación integral.

\section{Tabla 5}

Estadígrafos de las variables de estudio con sus respetivas dimensiones.

\begin{tabular}{lcccc}
\hline \hline & Media & Mediana & Moda & $\begin{array}{c}\text { Desv. } \\
\text { Desviación }\end{array}$ \\
\hline \hline D1. Clasificar metas de aprendizaje & 9,0357 & 9,0000 & $9,00^{a}$ & 2,23577 \\
D2. Obtener evidencias & 9,0714 & 9,0000 & $9,00^{a}$ & 2,35590 \\
D3. Retroalimentación & 8,8571 & 9,0000 & 9,00 & 223961 \\
D4. Estudiantes como dueños de su & 8,6786 & 9,0000 & 9,00 & 2,31026 \\
aprendizaje & & & & \\
D5. Estudiantes como recursos de & 9,0000 & 9,0000 & 12,00 & 2,43432 \\
aprendizaje. & 44,6429 & 42,0000 & 60,00 & 9,67323 \\
Variable 1: Evaluación formativa & 11,4643 & 12,0000 & 12,00 & 2,72821 \\
Dimensión humana & 11,1071 & 11,0000 & $8,00^{a}$ & 2,67137 \\
Dimensión intelectual & 10,8571 & 11,0000 & 11,00 & 2,67657 \\
Dimensión axiológica & 10,7857 & 10,0000 & 10,00 & 2,49974 \\
Dimensión social & 43,8214 & 43,0000 & 44,00 & 9,23381 \\
Variable 2: Formación integral & & & & \\
\hline \hline
\end{tabular}

Nota. Data de resultados obtenidos.

En la Tabla se muestra un estadístico de dispersión (desviación estándar) y medidas de tendencia central (Media, mediana, moda) que servirán de línea base para evaluar los cambios surgidos por la implementación de las recomendaciones hechas en la presente investigación. 


\section{Tabla 6}

Prueba de normalidad de Shapiro-Wilk de las variables evaluación formativa y formación integral con sus respectivas dimensiones.

\begin{tabular}{|c|c|c|c|c|c|c|}
\hline & \multicolumn{3}{|c|}{ Kolmogorov-Smirnov ${ }^{a}$} & \multicolumn{3}{|c|}{ Shapiro-Wilk } \\
\hline & Estadístico & $\mathrm{gl}$ & Sig. & Estadístico & gl & Sig. \\
\hline $\begin{array}{l}\text { Variable 1: Evaluación } \\
\text { formativa }\end{array}$ & ,200 & 28 & ,006 & ,889 & 28 & ,006 \\
\hline $\begin{array}{l}\text { Variable 2: Formación } \\
\text { integral }\end{array}$ & ,207 & 28 & ,003 & ,886 & 28 & ,005 \\
\hline
\end{tabular}

Nota. Data de resultados obtenidos

En la tabla se observa que la cantidad de los sujetos muéstrales que intervienen en la investigación es menor de 50, por lo cual se decide utilizar el juicio de normalidad de ShapiroWilk en donde se observa que los sig. Bilateral son en su mayoría menores de 0,05 por lo cual orienta a usar la correlación de Rho de Spearman para la comprobación de la prueba de hipótesis en el apartado correspondiente.

\section{Comprobación de la hipótesis general}

Hipótesis nula - Ho: La evaluación formativa no influye significativamente en la formación integral de los estudiantes de la Institución Educativa № 80055 "Juan Ignacio Gutiérrez Fuente", Macabí Bajo, Paiján, 2020.

Hipótesis alterna - Hi: La evaluación formativa influye significativamente en la formación integral de los estudiantes de la Institución Educativa Nº 80055 “Juan Ignacio Gutiérrez Fuente", Macabí Bajo, Paiján, 2020.

\section{Tabla 7}

Correlación de Rho de Spearman entre evaluación formativa y formación integral.

\begin{tabular}{lllr}
\hline \hline & & $\begin{array}{c}\text { Variable 2: Formación } \\
\text { integral }\end{array}$ \\
\hline Rho de Spearman & Variable 1: Evaluación & Coeficiente de &, $737^{* *}$ \\
& formativa & correlación &, 000 \\
& & Sig. (bilateral) & 28 \\
& $N$ & \\
\hline
\end{tabular}

Nota. Data de resultados obtenidos.

Nivel de significación

$$
\text { : } \alpha=0,05 \text { (prueba bilateral) }
$$


Valor de Rho Spearman calculado

: 0,737

Valor de $\mathrm{P}$

: 0,001

El valor de Rho Spearman= 0,737 y el significado bilateral obtenido es 0,001 valor que es buena a la región crítica $\alpha=0,05$; en consecuencia, se rechaza la hipótesis nula y se acepta la hipótesis alterna. Por lo tanto, se concluye que la evaluación formativa influye significativamente en la formación integral de los estudiantes de la Institución Educativa $N^{\circ}$ 80055 “Juan Ignacio Gutiérrez Fuente”, Macabí Bajo, Paiján, 2020.

\section{DISCUSIÓN}

En la presente investigación se indagó sobre las variables de estudio evaluación formativa y formación integral de los estudiantes de la Institución Educativa № 80055 "Juan Ignacio Gutiérrez Fuente”, Macabí Bajo, Paiján, 2020; para lo cual se aplicó cuestionarios que permitieron recoger datos, los cuales fueron construidos teniendo en cuenta sus dimensiones que permitieron conocer las opiniones de la muestra de estudio para luego procesarse y construir tablas, figuras y realizar la prueba de hipótesis. A continuación, se procede a realizar el proceso de la triangulación de la discusión:

La dimensión clasificar metas de aprendizaje influye significativamente en la formación integral de los estudiantes de la Institución Educativa № 80055 "Juan Ignacio Gutiérrez Fuente", Macabí Bajo, Paiján, 2020. Obteniéndose un Rho Spearman= 0,456 y el significado bilateral obtenido es 0,015 valor que es inferior a la región crítica $\alpha=0,05$; en consecuencia, se rechaza la hipótesis nula y se acepta la hipótesis alterna. Dichos resultados se corroboran con lo planteado por Castillo (2017) concluye que existe relación positiva entre las variables de estudio de $\mathrm{t=}=093$ por lo cual se rechaza la hipótesis nula y se acepta alterna, lo mismo sucede con las hipótesis específicas planteadas. Se sustenta con lo planteado por William (2011) quien se refiere al proceso de la presentación, aclaración y comprensión de los propósitos de aprendizaje que deben de adquirir los estudiantes para la construcción de las competencias según lo planteado en el currículo nacional; teniendo en cuenta que para la obtención de las metas es necesario tener preciso que se necesita tener claro lo que se quiere lograr. Además, consiste en realizar un conjunto de acciones para realizar el proceso de recopilación de la información entendible y manejable que pueda ser empleada para 
mejorar el proceso de enseñanza.

La dimensión obtener evidencias influye significativamente en la formación integral de los estudiantes de la Institución Educativa N 80055 “Juan Ignacio Gutiérrez Fuente”, Macabí Bajo, Paiján, 2020. Obteniéndose un Rho Spearman= 0,583 y el significado bilateral obtenido es 0,001 valor que es inferior a la región crítica $\alpha=0,05$; en consecuencia, se rechaza la hipótesis nula y se acepta la hipótesis alterna, los resultados se corroboran con lo obtenido por Martínez (2016) quien concluye que se debe comprender que la escuela es la parte fundamental dentro de una sociedad, la cual requiere de un proceso dinamizador que permita la construcción de aprendizajes significativos. Se sustenta con lo planteado por William (2011) quien señala que consiste en realizar un conjunto de acciones para realizar el proceso de recopilación de la información entendible y manejable que pueda ser empleada para mejorar el proceso de enseñanza.

La dimensión retroalimentación influye significativamente en la formación integral de los estudiantes de la Institución Educativa № 80055 “Juan Ignacio Gutiérrez Fuente”, Macabí Bajo, Paiján, 2020. Obteniéndose un Rho Spearman= 0,622 y el significado bilateral obtenido es 0,001 valor que es inferior a la región crítica $\alpha=0,05$; en consecuencia, se rechaza la hipótesis nula y se acepta la hipótesis alterna. Dichos resultados se corroboran con lo planteado por Médico (2016) quien concluye que la metodología empleada con los estudiantes asociada a la ABP y la evaluación formativa son parte fundamental para la formación integral de los estudiantes, a través de un proceso enriquecedor lleno de aprendizajes. Se sustenta con lo planteado por William (2011) se refiere al proceso de recoger de los estudiantes sus evidencias de aprendizaje para determinar cuál es su desenvolvimiento en las actividades asignadas para poder realizar el proceso de autorregulación donde se produzca la reflexión y la mejora según las observaciones realizada por el docente.

La dimensión, estudiantes como dueños de su aprendizaje, influye significativamente en la formación integral de los estudiantes de la Institución Educativa № 80055 "Juan Ignacio Gutiérrez Fuente", Macabí Bajo, Paiján, 2020. Obteniéndose un Rho Spearman= 0,550 y el significado bilateral obtenido es 0,002 valor que es inferior a la región crítica $\alpha=0,05$; en 
consecuencia, se rechaza la hipótesis nula y se acepta la hipótesis alterna. Dichos resultados se corroboran con lo obtenido por Vera (2019) quien concluye que, existe una relación positiva de $\mathrm{Rho}=0,920$ __ valor $=0,000<0,005$ por lo tanto, se determina que, la evaluación formativa tiene una relación significativa en la elaboración de textos de manera escrita. Se sustenta con lo planteado por William (2011) quien lo considera como un aspecto fundamental dentro del proceso de evaluación formativa porque en ellos se centra el desarrollo de la enseñanza según los objetivos propuestos. Para observar dicho protagonismo es necesario que convierta en el constructor de su propio aprendizaje a través de un protagonismo activo dentro de las aulas de clase.

La dimensión, estudiantes como recursos de aprendizajes, influye significativamente en la formación integral de los estudiantes de la Institución Educativa № 80055 "Juan Ignacio Gutiérrez Fuente", Macabí Bajo, Paiján, 2020. Obteniéndose un Rho Spearman= 0,616 y el significado bilateral obtenido es 0,000 valor que es inferior a la región crítica $\alpha=0,05$; en consecuencia, se rechaza la hipótesis nula y se acepta la hipótesis alterna. Dichos resultados se corroboran con lo obtenido por Pacheco (2019) quien concluye que existe una relación positiva y alta entre las variables de estudio de Rho de Spearman es 0.786 y el $p=0.000$, lo cual representa que coexiste una elevada concordancia afirmativa. Se sustenta con lo planteado por William (2011) se traduce como los agentes en donde el docente construye competencias y por lo cual lo considera como recursos, además se convierten en un medio de aprendizaje cuando comparten sus saberes con sus compañeros.

La evaluación formativa influye significativamente en la dimensión humana de los estudiantes de la Institución Educativa № 80055 “Juan Ignacio Gutiérrez Fuente”, Macabí Bajo, Paiján, 2020. Obteniéndose un Rho Spearman= 0,644 y el significado bilateral obtenido es 0,000 valor que es inferior a la región crítica $\alpha=0,05$; en consecuencia, se rechaza la hipótesis nula y se acepta la hipótesis alterna. Dichos resultados se corroboran con lo obtenido por Joya (2019) quien concluye que el currículo que brinda actualmente Ministerio de Educación, lo cual es un desafío para los docentes porque se cetra en un enfoque por competencias y se trabaja en base a una evaluación formativa. Se sustenta con lo planteado por MINEDU (2013) quien señala que la dimensión humana consiste en desarrollar las capacidades, virtudes y actitudes que están relacionadas con llevar una vida que respeta la 
condición humana y el entorno social donde se desenvuelve, es necesario saber que el ser humano presenta dos facultades que lo diferencian de los animales irracionales, las cuales son la inteligencia para decidir y actuar correctamente y la voluntad que los hacen personas racionales y superiores.

La evaluación formativa influye significativamente en la dimensión intelectual de los estudiantes de la Institución Educativa № 80055 “Juan Ignacio Gutiérrez Fuente”, Macabí Bajo, Paiján, 2020 y los objetivos específicos son los siguientes. Obteniéndose un Rho Spearman $=0,693$ y el significado bilateral obtenido es 0,001 valor que es inferior a la región crítica $\alpha=0,05$; en consecuencia, se rechaza la hipótesis nula y se acepta la hipótesis alterna. Dichos resultados se corroboran con lo obtenido por Rodríguez (2017) quien concluye que, nivel de satisfacción en cuanto al perfil del docente y el sistema de evaluación que se emplea se ubican en el nivel de satisfactorio. Asimismo, existe un adecuado nivel en el empleo de estrategias para la formación integral de los educandos. Se sustenta con lo planteado por Luzuriaga (1960) está traducida en el desarrollo del pensamiento lógico, critico, analítico y creativo que le permita la obtención de aprendizaje significativos y de tener una autoformación a lo largo de su vida; de esta manera el estudiante es competente, adquiere habilidades para razonar, analizar, argumentar, entre otros que le permita generar nuevos conocimientos y brindar soluciones creativas y precisas a los problemas que se presentan.

La evaluación formativa influye significativamente en la dimensión axiológica de los estudiantes de la Institución Educativa № 80055 “Juan Ignacio Gutiérrez Fuente”, Macabí Bajo, Paiján, 2020. Obteniéndose un Rho Spearman= 0,780 y el significado bilateral obtenido es 0,001 valor que es inferior a la región crítica $\alpha=0,05$; en consecuencia, se rechaza la hipótesis nula y se acepta la hipótesis alterna. Dichos resultados se corroboran con lo obtenido por Paredes (2019) quien concluye que existe una relación positiva de Rho= 0.630 entre las variables de estudio, lo cual nos demuestra que una guarda relación con la otra pero que existen otros factores que alteran su comportamiento. Así mismo, existe una relación alta entre las hipótesis especificas planteadas. Se sustenta con lo planteado por Sánchez y Castillejo (1983) quien sostiene que la dimensión axiológica es necesario que se adquieran valores que construyan su personalidad, les permita construir sus percepciones 
y tomar decisiones; es necesario sostener que los seres humanos somos diferentes, por ende, es necesario que nos respetemos para tener una mejor convivencia.

La evaluación formativa influye significativamente en la dimensión social de los estudiantes de la Institución Educativa № 80055 “Juan Ignacio Gutiérrez Fuente”, Macabí Bajo, Paiján, 2020 y los objetivos específicos son los siguientes. Obteniéndose un Rho Spearman= 0,688 y el significado bilateral obtenido es 0,001 valor que es inferior a la región crítica $\alpha=0,05$; en consecuencia, se rechaza la hipótesis nula y se acepta la hipótesis alterna. Dichos resultados se corroboran con lo obtenido por Alva (2018) quien concluye que existe una relación significativa de Rho de Spearman $=0,675$ lo cual demuestra que ambas variables si se relacionan, donde el nivel formación profesional en los estudiantes es de un 93\%. Se sustenta con lo planteado por Lezurriaga (1960) quien señala que la dimensión social, el ser humano necesita fortalecer sus valores y actitudes que le permitan respetar y valorar al otro; por eso, es necesario que el ser humano sea sensible, reconozca las problemáticas sociales para brindarle soluciones a través de trabajos de manera individual o grupal, respetando opiniones y la diversidad cultural.

La evaluación formativa influye significativamente en la formación integral de los estudiantes de la Institución Educativa № 80055 “Juan Ignacio Gutiérrez Fuente”, Macabí Bajo, Paiján, 2020. Obteniéndose un Rho Spearman $=0,737$ y el significado bilateral obtenido es 0,001 valor que es inferior a la región crítica $\alpha=0,05$; en consecuencia, se rechaza la hipótesis nula y se acepta la hipótesis alterna. Dichos resultados se corroboran con lo obtenido por Castillo (2017) concluye que existe relación positiva entre las variables de estudio de $\mathrm{\tau}=, 093$ por lo cual se rechaza la hipótesis nula y se acepta alterna, lo mismo sucede con las hipótesis específicas planteadas. Se sustenta con lo planteado por el MINEDU (2016) que señala que la evaluación formativa según el CNEB es un proceso que tiene como objetivo garantizar la formación integral del estudiante a través de acompañamiento sistemático, regulado y persistente se logra lo planteado teniendo en cuenta los objetivos educativos que se desarrolla a través de acciones que va más allá de la obtención de solo calificaciones, dejando de lado lo correcto e incorrecto sino la reflexión de lo obtenido y Paiba (2007) sostiene que la formación integral se traduce cuando se desarrollan todas las dimensiones en un ser humano (cognitivo, actitudinal, habilidades y valores) empezando desde el aspecto 
intelectual a través de la construcción de aprendizajes, el aspecto humano adquiriendo valores morales y la ética y el aspecto social para realizar una interacción saludable.

\section{CONCLUSIONES}

La evaluación formativa influye significativamente en la formación integral de los estudiantes de la Institución Educativa № 80055 “Juan Ignacio Gutiérrez Fuente”, Macabí Bajo, Paiján, 2020. Lo cual se sustenta en un Rho Spearman $=0,737$ y el significado bilateral obtenido es 0,001 valor que es inferior a la región crítica $\alpha=0,05$; en consecuencia, se rechaza la hipótesis nula y se acepta la hipótesis alterna.

La dimensión clasificar metas de aprendizaje influye significativamente en la formación integral de los estudiantes de la Institución Educativa N 80055 "Juan Ignacio Gutiérrez Fuente", Macabí Bajo, Paiján, 2020. Lo cual se sustenta en un Rho Spearman= 0,456 y el significado bilateral obtenido es 0,015 valor que es inferior a la región crítica $\alpha=0,05$; en consecuencia, se rechaza la hipótesis nula y se acepta la hipótesis alterna.

La dimensión obtener evidencias influye significativamente en la formación integral de los estudiantes de la Institución Educativa № 80055 "Juan Ignacio Gutiérrez Fuente”, Macabí Bajo, Paiján, 2020. Lo cual se sustenta en un Rho Spearman= 0,583 y el significado bilateral obtenido es 0,001 valor que es inferior a la región crítica $\alpha=0,05$; en consecuencia, se rechaza la hipótesis nula y se acepta la hipótesis alterna.

La dimensión retroalimentación influye significativamente en la formación integral de los estudiantes de la Institución Educativa № 80055 "Juan Ignacio Gutiérrez Fuente”, Macabí Bajo, Paiján, 2020. Lo cual se sustenta en un Rho Spearman= 0,622 y el significado bilateral obtenido es 0,000 valor que es inferior a la región crítica $\alpha=0,05$; en consecuencia, se rechaza la hipótesis nula y se acepta la hipótesis alterna.

La dimensión, estudiantes como dueños de su aprendizaje, influye significativamente en la formación integral de los estudiantes de la Institución Educativa № 80055 "Juan Ignacio Gutiérrez Fuente”, Macabí Bajo, Paiján, 2020. Lo cual se sustenta en un Rho Spearman= 0,550 y el significado bilateral obtenido es 0,002 valor que es inferior a la región crítica $\alpha=$ 
0,05; en consecuencia, se rechaza la hipótesis nula y se acepta la hipótesis alterna.

La dimensión, estudiantes como recursos de aprendizajes, influye significativamente en la formación integral de los estudiantes de la Institución Educativa Nº 80055 "Juan Ignacio Gutiérrez Fuente", Macabí Bajo, Paiján, 2020. Lo cual se sustenta en un Rho Spearman= 0,616 y el significado bilateral obtenido es 0,001 valor que es inferior a la región crítica $\alpha=$ 0,05; en consecuencia, se rechaza la hipótesis nula y se acepta la hipótesis alterna.

La evaluación formativa influye significativamente en la dimensión humana de los estudiantes de la Institución Educativa N 80055 “Juan Ignacio Gutiérrez Fuente”, Macabí Bajo, Paiján, 2020. Lo cual se sustenta en un Rho Spearman= 0,644 y el significado bilateral obtenido es 0,000 valor que es inferior a la región crítica $\alpha=0,05$; en consecuencia, se rechaza la hipótesis nula y se acepta la hipótesis alterna.

La evaluación formativa influye significativamente en la dimensión intelectual en los estudiantes de la Institución Educativa № 80055 “Juan Ignacio Gutiérrez Fuente”, Macabí Bajo, Paiján, 2020. Lo cual se sustenta en un Rho Spearman= 0,693 y el significado bilateral obtenido es 0,000 valor que es inferior a la región crítica $\alpha=0,05$; en consecuencia, se rechaza la hipótesis nula y se acepta la hipótesis alterna.

La evaluación formativa influye significativamente en la dimensión axiológica de los estudiantes de la Institución Educativa Nº 80055 “Juan Ignacio Gutiérrez Fuente”, Macabí Bajo, Paiján, 2020. Lo cual se sustenta en un Rho Spearman= 0,780 y el significado bilateral obtenido es 0,001 valor que es inferior a la región crítica $\alpha=0,05$; en consecuencia, se rechaza la hipótesis nula y se acepta la hipótesis alterna.

La evaluación formativa influye significativamente en la dimensión social de los estudiantes de la Institución Educativa N 80055 “Juan Ignacio Gutiérrez Fuente”, Macabí Bajo, Paiján, 2020. Lo cual se sustenta en un nivel medio de la dimensión social de la formación integral; además se ha obtenido un Rho Spearman= 0,688 y el significado bilateral obtenido es 0,001 valor que es inferior a la región crítica $\alpha=0,05$; en consecuencia, se rechaza la hipótesis nula y se acepta la hipótesis alterna. 


\section{REFERENCIAS}

Alva, G. (2018) Educación religiosa y formación integral de los estudiantes del nivel secundaria de la institución educativa "Simón Bolívar", Otuzco - 2017, Universidad César Vallejo.

Amilburu, M. (2010) Aprendiendo a ser humanos. Una Antropología de la Educación, Eunsa, Pamplona.

Brookhart, S. (2003). Developing Measurement Theory for Classroom Assessment Purposes and Uses. Educational Measurement: Issues and Practice, 22 (4), 5-12.

Castillo, J. (2017) Influencia del Manual de Convivencia en la formación integral de los estudiantes del Colegio de Alto Rendimiento de La Libertad, 2017, Universidad César Vallejo.

Condemarín, M. y Medina, A. (2012). Evaluación de los aprendizajes, un medio mejorar las competencias lingüísticas y comunicativas. Santiago de Chile, Mineduc (P900).

Joya M. (2019) La evaluación formativa desde la práctica docente en la Institución Educativa Sor Querubina de San Pedro de Surquillo, 2018, Universidad César Vallejo.

Martínez, I. (2016) "Formación integral y complejidad en la educación básica secundaria”, Universidad Simón Bolívar, Colombia.

Maslow, A. H. "A theory of human motivation”. Psychological Review 50. 1943: 370-396.

Médico, E. (2016) La evaluación formativa al interior del espacio de tutoría: Sentidos, implicancias y potencialidades que sus actores les otorgan en el 3er. año del Plan de Estudios de la carrera de Medicina de la UNL, Argentina (Universidad Nacional del Litoral).

Ministerio de educación. (2016). Currículo nacional de la Educación Básica.

Pacheco, L. (2019) Evaluación formativa y el aprendizaje por competencias en el área de ciencia y tecnología de los estudiantes de quinto grado de nivel secundaria de la institución educativa Nº 6016 Jesús Salvador UGEL 01 - Pachacamac, año - 2019, Universidad César Vallejo.

Paiba (2007) sostiene que se traduce cuando se desarrollan todas las dimensiones en un ser humano (cognitivo, actitudinal, habilidades y valores).

Paredes, C. (2019) Gestión tutorial y formación integral del estudiante de educación inicial de la Institución Educativa "Salaverry"- 2019; Universidad César Vallejo.

Pigott, T., y Polanin, J. (2011). Educational assessment. Oxford: Oxford University Press. 
Rodríguez, M. (2017) "Evaluación de un currículo centrado en la formación integral de los estudiantes", Universidad Católica, Colombia.

Rodríguez, T. (2017) Función tutorial y formación integral del estudiante en la institución educativa privada internacional Elim de Tacna-2017, Universidad César Vallejo.

Sadler, R. (1989). Formative assessment and the design of instructional systems. Instructional Science, 18, 119---144.

Scriven, Michael. (1967). "The metodology of evaluation", en Perspectives of curriculum evaluation. Chicago: Rand McNally

Shepard, L. (2006). La evaluación en el aula. México: Instituto Nacional para la Evaluación de la Educación.

Velasquez, A. y Rey, N. (1999). Metodología de la investigación científica. Lima: Editorial San Marcos.

Vera, J. (2019) Evaluación formativa y la producción de textos escritos de los estudiantes del $\vee$ ciclo de la I.E. No 20547 "Manuel A. Odría" de la Ugel 15 - Huarochirí, Universidad César Vallejo.

Wiliam, D. (2011). Embedded formative assessment. United Stated of America: Solution Tree. 